Rev. Elev. Méd. vét. Pays trop., 1973, 26 (4) : 1 a - 4 a

\title{
Le droit de la mer et l'utilisation rationnelle des ressources halieutiques
}

\author{
par I. M. DIA (*)
}

\begin{abstract}
RESUME
L'auteur expose l'état actuel de l'exploitation des ressources de la mer sur la Côte africaine. Il évoque la nécessité d'établir de nouvelles règles de droit maritime intemational en vue de préserver les stocks de poissons tout en tenant compte des intérêts des pays participant à la pêche.
\end{abstract}

L'importance des ressources halieutiques dans la nourriture des populations des pays en voie de développement et dans l'accélération des processus de développement industriel de ces pays trouve son illustration dans le volume des investissements qui ont été consentis à leur exploitation au cours de cette dernière décade.

Les statistiques récemment publiées par la FAO font état d'un coût global des investissements dans le secteur de la pêche de 6380 millions de dollars, soit l'équivalent de 1500 milliards de francs CFA dans les seuls pays atlantiques d'Afrique entre 1960 et 1970.

L'évolution des techniques et l'amélioration des possibilités des pays en voie de développement se traduiront par des investissements encore plus importants d'autant que l'accroissement de la population continuera de motiver la recherche d'une quantité plus importante de protéines animales.

Le problème se pose alors d'un aménagement des ressources de la mer qui puisse rationaliser leur exploitation et prévenir le danger d'un dépeuplement.

Les connaissances actuelles sur limportance des stocks halieutiques et leur répartition, en ce qui concerne l'Atlantique le long des côtes africaines, conduisent en tout cas à la conclusion qu'il est devenu urgent et impérieux de les protéger pour éviter leur destruction.

Le long de cette côte les ressources sont inégalement réparties. Elles sont abondantes dans les zones où se produisent les phénomènes de remontée en surface de masses d'eaux profondes chargées d'éléments nutritifs. Elles sont clairsemées ou inexistantes ailleurs.

Les principales zones de ces mouvements sont identifiées. Il s'agit, pour l'Atlantique central et oriental de la partie nord de Sierra Leone jusqu'au Cap Spartel et de l'embouchure du Congo au Cap de Bonne Espérance.

Ces phénomènes de remontées déterminent, dans une certaine mesure, la richesse halieutique et la diversité faunistique qui est variable selon les aires.

(₹) Docteur Vétérinaire, Directeur de l’Océanographie et des Pêches Maritimes, B.P. 289, Dakar - Sénégal. 
Dans la partie nord de l'Atlantique du Centre Est qui intéresse les pays d'Afrique occidentale, d'importants stocks de sardines, merlus, sparidae, crevettes, langoustes, chinchards, maquereaux, sardinelles et thons sont signalés, dont on estime l'importance à 600000 tonnes pour les stocks pélagiques.

Le long des côtes du Golfe de Guinée, on rencontre principalement des espèces pélagiques mais aussi des merous, des brêmes et des crevettes de diverses variétés qui s'étagent sur des profondeurs variables selon les espèces.

Les estimations permettent de chiffrer les apports à 1,5 million de tonnes pour les stocks demersaux et près de 5 millions pour les espèces pélagiques.

Un autre élément important à retenir est la répartition bathymétrique des espèces. La division, habituellement adoptée en espèces pélagiques et espèces demersales est déjà une indication et. une référence bathymétrique. Elle n'est pas absolue. C'est ainsi que les nouvelles techniques modernes permettent de capturer des sardinelles de grosse taille, au chalut pélagique, par les fonds de 1500 à 2000 mètres. Certains stocks de crevettes sont pêchés par les fonds de 600 à 800 mètres.

Il faut noter que la plupart des espèces suivent un mouvement migratoire dont l'amplitude et le sens sont variables et que ces mouvements les conduisent à une répartition qui dépasse la répartition actuellement connue et qui fixe, en gros, trois sortes de peuplements :

- un peuplement de la première zone du plateau continental où l'on trouve des pélagiques côtiers comme les sardinelles, les chinchards, maquereaux : des espèces demersales comme les sparidae, les crevettes, les langoustes et autres qui se rencontrent de la côte aux profondeurs de 200 mètres;

- un peuplement de la $2^{e}$ zone du plateau correspondant à la pente continentale jusqu'aux fonds de 800 à $1000 \mathrm{~m}$ où l'on trouve d'importantes quantités de crustacés et poissons industriels;

- un peuplement pélagique hauturier constitué essentiellement par les grands thonidae que l'on trouve sur la marge de la pente.

Cette répartition est schématique. Elle recouvre la réalité d'une certaine expérience mais ne tient pas compte des corrélations de milieu et également de l'action des courants marins. Elle a l'avantage de démontrer l'erreur généralement commise lorsqu'il s'agit de fixer par la distance les aires de répartition des espèces en décidant que la zone de souveraineté d'un Etat s'exerce sur un nombre défini de milles marins à partir de son rivage.

En fait, la considération des données de la biologie des espèces permet de dire au contraire que le critère géomorphologique de la profondeur est sans aucun doute le moins incertain pour fixer l'aire de dispersion des espèces exploitables et par conséquent la limite à laquelle la souveraineté d'un Etat devrait s'étendre pour assurer une protection efficace de ses ressources halieutiques.

Pour la partie de l'Atlantique central qui baigne les côtes d'Afrique, les principales ressources, en dehors des thonidae, résident dans la partie du plateau continental entre le rivage et l'isobathe des 600 mètres.

\section{DROIT INTERNATIONAL ET POLITIQUE DES PECHES}

Ces considérations sont pour les pays situés le long de l'Atlantique central les bases de leurs politiques de pêche.

Celles-ci visent en général deux objectifs qu découlent l'un de l'autre :

- le premier est d'assurer l'approvisionnement des pays en poissons divers soit par l'accroissement des flottes nationales soit par l'importation à partir de flottes étrangères;

- le second est d'assurer la fixation d'une industrie de pêche créatrice de revenus supplé- 
mentaires par sa valeur ajoutée et d'emplois, en même temps qu'il transforme la position du pays qui, d'importateur veut devenir exportateur de produits halieutiques.

Dans cette démarche, les pays ont conscience de leur force qui résulte de leur position par rapport aux lieux de pêche et de leur faiblesse qui est la conséquence de leur sous-équipement. Ils trouvent donc dans l'extension de leur juridiction sur des étendues de la haute mer adjacente à leurs côtes, un élément d'appui pour cette politique d'autant plus que les flottes étrangères opèrent sans contrepartie dans ces zones et qu'à terme, un grave danger menace les stocks si des mesures de protection concertées ne sont pas mises en application à brève échéance.

En 1958, le total des captures du Cap Spartel à l'embouchure du Congo se chiffrait à 390000 tonnes. Pour 28 pays qui ont participé à la pêche, 5 étaient des pays africains riverains.

En 1969 , ce tonnage est passé à 1960000 tonnes pour 38 pays participants dont 17 non africains. Pour ce tonnage, les pays riverains avaient réalisé par leurs propres moyens 732000 tonnes, soit 32 p. 100 seulement des prises !

La politique des Etats se heurte à la notion de droit international maritime, notion évoquée par les pays dont les flottes opèrent dans ces zones. Celui-ci est constitué par un certain nombre de textes de portée générale, relativement diffus et non reconnus par tous les Etats.

Parmi les plus célèbres de ces textes, on peut citer les 4 conventions de Genève adoptées en avril 1958 et soumises à la ratification des Etats :

- la Convention sur la haute mer;

- la Convention sur la pêche et la préservation des ressources biologiques de la mer;

- la Convention sur le plateau continental;

- la Convention sur la mer territoriale et la zone contiguë.

L'économie générale de ces textes était d'instituer une mer territoriale de douze milles marins, prolongement des Etats riverains et dans laquelle chaque Etat exercerait toutes compétences de juridiction pour la pêche comme pour le reste. Les ressources minérales du plateau continental appartiennent aux Etats mais les ressources naturelles leur échappent, du moins celles qui sont situées hors d'une mer territoriale fixée à 12 milles.

En fait, ce droit international n'est pas accepté universellement et c'est ce qui est à la base de la décision de la $25^{\mathrm{e}}$ Assemblée générale des Nations unies de convoquer une nouvelle conférence sur le droit de la mer en 1974.

En effet, vis-à-vis des Conventions de Genève de 1958, on peut considérer que les Etats du monde se répartissent selon le tableau ci-après pour le problème de la mer territoriale :

- 27 Etats continuent d'adopter 3 milles marins;

- 17 Etats revendiquent entre 3 et 12 milles marins;

- 51 Etats ont 12 milles;

- 6 Etats ont entre 12 et 200 milles;

- 9 Etats revendiquent 200 milles.

En ce qui concerne l'exploitation et l'utilisation des ressources de la pêche, la divergence d'opinion a dépassé la simple controverse académique pour se situer sur un plan économique et opposer les pays développés et les pays en voie de développement.

Les premiers se réfèrent à la Convention de Genève de 1958 et cherchent à limiter la souveraineté nationale à une étendue de 12 milles, le reste étant ouvert à toutes les nations, au nom du droit international. Certains acceptent de discuter certains droits préférentiels avec les pays riverains sur des étendues plus importantes de la haute mer mais guère plus.

Les seconds, du moins pour les plus violents, partent du principe que la limite de la mer territoriale doit être fixée en fonction de conditions géographiques et des intérêts économiques 
de chaque nation dune façon souveraine. Ce principe, affirmé dans la Déclaration de Lima, aboutira à l'adoption d'une limite de la mer territoriale de 200 milles pour de nombreux Etats latino-américains.

Dans ce dialogue de sourds, il importe de noter qu'en fait le problème des eaux territoriales ne s'assimile pas totalement à celui du régime d'exploitation des eaux en matière de pêche et que l'origine du conflit procède de l'assimilation abusive de deux concepts qui ne s'identifient pas :

- le concept d'eau territoriale a une inspiration militaire et de défense;

- celui de zone de pêche, beaucoup plus économique, recherche à établir pour un pays déterminé des espaces plus étendus sur la haute mer pour permettre une exploitation quasi exclusive pour ce pays des ressources qui y sont contenues. Si la discussion peut être ouverte sur le régime des eaux territoriales à cause des implications qu'entraînent leur extension abusive, par contre il est tout à fait juste, face à l'accroissement de Ia flotte internationale qui, à terme finira par poser bien des problèmes aux économies des Etats riverains, que ces Etats envisagent des mesures de sauvegarde pour assurer la préservation des stocks en vue de l'aménagement rationnel de leur exploitation.

C'est sans doute vers ce compromis qu'il faudra orienter la recherche d'un droit maritime nouveau qui tiendra compte des intérêts généraux de toute la communauté internationale et qui s'attachera à sauvegarder le patrimoine commun que représentent les ressources de la mer.

\section{SUMMARY}

\section{Sea right and the rational use of halieutic resources}

The author shows the actual condition of the explotation in sea resources on the African coast.

$\mathrm{He}$ focuses the necessity to establish new rules of international maritime right in order to preserve the stocks of fishes but taking into consideration the interests of the countries which participate to the fishing.

\section{RESUMEN}

El derecho del mar y la utilización racional de los recursos de la pesca

EI autor expone el estado actual de la explotación de los recursos del mar a lo largo de la costa africana. Eyoca la necesidad de establecer nuevas reglas de derecho maritimo internacional para preservar las existencias de peces al tener cuenta de los intereses de los paises participando en la pesca. 